\title{
Composição e caracterização da fauna de mamíferos do Parque Nacional das Emas, Goiás, Brasil
}

\author{
Flávio H.G. Rodrigues ${ }^{1}$ \\ Leandro Silveira ${ }^{1}$ \\ Anah T.A. Jácomo ${ }^{1}$ \\ Ana Paula Carmignotto ${ }^{2}$ \\ Alexandra M.R. Bezerra ${ }^{3}$ \\ Daniela Cunha Coelho 4 \\ Hamilton Garbogini ${ }^{5}$ \\ Juliana Pagnozzi ${ }^{2}$ \\ Adriani Hass ${ }^{6}$
}

\begin{abstract}
Composition and characterization of the mammal fauna of Emas National Park, Goiás, Brasil. Emas National Park is a very important Conservation Unit in the Cerrado Biome. Nevertheless the fauna of this region is still poorly known. In this study, a revised list of the mammal fauna of the Emas National Park with comments about the composition and the number of species surveyed is presented. Eighty six species of mammals were recorded in the Park and neighboring area, including several rare and threatened species, like the bush dog (Speothos venaticus (Lund, 1842)), Marsh deer (Blastocerus dichotomus (Illiger, 1815)) and others. The giant rat (Kunsia tomentosus (Lichtenstein, 1830)) was recorded for the first time in a conservation unit. On the other hand, species usually very common in the Cerrado biome, don't occur in Emas National Park: the brazilian rabbit (Sylvilagus brasiliensis (Linnaeus, 1758)), marmosets (Callithrix spp.), and the punaré (Thrichomys apereoides (Lund, 1841)).

KEY WORDS. Cerrado, conservation, mammalian survey
\end{abstract}

O Cerrado, segundo maior bioma sul-americano, abrange mais de dois milhões de quilômetros quadrados e possui uma rica fauna de mamíferos composta por 190 espécies (MARINHO-FILHO et al., no prelo). Ao longo dos últimos 50 anos o Cerrado vem sendo gradativamente substituído por áreas de pastagem e lavoura (NEPSTAD et al. 1997) e hoje 35\% da área total do Bioma já está ocupado por atividades agrícolas (MACEDO 1995). Atualmente menos de 1\% do Cerrado está preservado em unidades de conservação federais de uso indireto (PÁDUA 1996;

1) Associação Pró-Carnívoros. SQN 412 K 305, 70867-110 Brasília, Distrito Federal. E-mail: rodrigues@procarnivoros.org.br

2) Departamento de Mastozoologia, Museu de Zoologia, Universidade de São Paulo.

3) Departamento de Mastozoologia, Museu Nacional, Universidade Federal do Rio de Janeiro

4) Pós Graduação em Biologia Animal, Universidade de Brasília.

5) FURNAS Centrais Elétricas S.A.

6) Departamento de Ecologia, Universidade de Brasília. 
WWF 1999) e muitas destas áreas não estão suficientemente estruturadas para garantir a integridade do ecossistema local (WWF 1999). O Parque Nacional das Emas (PNE), devido sua extensão e integridade de habitats, representa uma das mais importantes unidades de conservação do Cerrado e é reconhecido internacionalmente por possuir uma fauna exuberante e de fácil observação (ERIZE 1977; REDFORD 1985a). No entanto, apesar de estudos terem sido realizados com algumas espécies de mamíferos de médio e grande porte (REDFORD 1985b; REDFORD 1987; RODRIGUES 1996; RODRIGUES \& MONTEIRO-FILHO 1997, 1999, 2000; RODRIGUES et al. 1999; SilveIRA et al. 1997, 1998, 1999), a fauna de mamíferos de pequeno porte do PNE permanece pouco conhecida. O único inventário de mamíferos realizado no PNE foi baseado em observações e entrevistas com moradores locais e contemplou apenas espécies de médio e grande porte, além de uma espécie de marsupial (REDFORD 1983). O objetivo deste trabalho é fornecer uma listagem e uma caracterização dos mamíferos do PNE, destacando informações relevantes sobre a situação local de algumas espécies.

\section{MATERIAL E MÉTODOS}

\section{Área de estudo}

O Parque Nacional das Emas abrange área de aproximadamente 132.000 ha (IBDF/FBCN 1981) e está localizado no extremo sudoeste do estado de Goiás, próximo às divisas com o Mato Grosso e Mato Grosso do Sul $\left(18^{\circ} 15^{\prime} 50^{\prime \prime} \mathrm{S}\right.$ e 5253'33"W, localização da sede). O PNE situa-se num planalto que é divisor de águas entre as bacias do Pantanal (rio Taquari), Araguaia (nascentes do rio Araguaia) e Paraná (para onde correm os dois rios que passam pelo PNE: Jacuba e Formoso).

As fisionomias vegetais encontradas no PNE são, seguindo a classificação de RIBEIRO \& WALTER (1998): mata de galeria inundável, mata de galeria não inundável, cerrado sensu stricto e suas variações de típico e ralo, veredas, campo sujo e limpo (com subtipos úmido, seco e de murundus). A fisionomia predominante é o campo sujo, que cobre mais de $70 \%$ do Parque e as matas são responsáveis por apenas 1,2 \% da cobertura vegetal (FERRONORTE 1998). A vegetação remanescente no entorno do Parque apresenta fitofisionomias diferentes de seu interior, com áreas de vegetação densa de cerrado sensu stricto e cerradão e maior quantidade de florestas. As formações campestres semelhantes às do PNE foram totalmente substituídas do entorno por agricultura, especialmente culturas de soja e milho.

\section{Método de amostragem}

Para amostrar os mamíferos presentes na área de estudo, diversas metodologias, descritas a seguir, foram utilizadas.

Pequenos mamíferos não voadores: utilizou-se armadilhas convencionais dos tipos "young" e "sherman", e armadilhas de queda ("pitfalls"), com esforço total de 10.664 armadilhas x noite (tipo "young" e "sherman") e 2.898 "pitfalls" x dia. Instalou-se as armadilhas convencionais ao longo de transectos, de forma a cobrir toda a variedade de ambientes disponível. Em cada ponto de captura utilizou-se, em média, 30 armadilhas espaçadas em torno de $15 \mathrm{~m}$ uma da outra, ao nível do solo, 
em sua maioria, e a uma altura de 1 a $2 \mathrm{~m}$, quando possível, nos ambientes que apresentaram estratificação vertical. Instalou-se os "pitfalls" em todos os habitats amostrados pelas armadilhas convencionais, dispostos em 30 estações de captura, cada qual composta por seis baldes de 35 litros na configuração de um T: três baldes instalados em linha e outros três perpendicularmente aos anteriores.

Realizou-se as coletas em quatro excursões de aproximadamente 15 dias: duas na estação chuvosa (novembro de 1998 e fevereiro de 1999) e duas durante a estação seca (maio e julho/agosto de 1999).

Mamíferos voadores: utilizou-se redes de neblina $(7 \times 3 \mathrm{~m})$ para captura de morcegos. Montou-se as redes em corredores e clareiras no interior de florestas, próximo a cursos d'água e perto a recursos alimentares utilizados por morcegos, como flores e frutos. Realizou-se um esforço de 1.278 redes x horas de amostragem. Realizou-se as capturas de morcegos nas mesmas datas citadas acima.

Mamíferos de médio e grande porte: realizou-se o inventário deste grupo através de observações diretas e indiretas (p.ex. rastros, fezes, tocas etc), de fevereiro de 1994 a agosto de 1999. Nesse período também foram realizados censos por transecto e capturas de algumas espécies com armadilhas tipo "tomahawk", em especial carnívoros. Animais encontrados mortos foram coletados quando possível.

Citogenética: coletou-se amostras de tecido de exemplares de pequenos mamíferos não voadores e voadores e as armazenou-se em etanol $70 \%$, para futuros estudos de DNA e proteínas. Além disso, realizou-se preparações citogenéticas que auxiliaram na identificação de algumas espécies. Obteve-se as preparações cromossômicas em campo a partir de medula óssea ou do baço. Além disso estabeleceu-se em laboratório culturas de fibroblastos da maioria das espécies de pequenos mamíferos não voadores.

Apesar de não ser possível uma comparação direta entre diferentes metodologias de amostragem, contabilizou-se o número de registros para cada espécie observada, para possibilitar a discussão sobre abundância relativa das espécies. A nomenclatura das espécies segue WILSON \& REEDER (1993) modificada segundo publicações mais recentes: BONVICINO \& ALMEIDA (2000), BONVICINO \& WEKSLER (1998), MUSSER et al. (1998), REDFORD \& EINSENBERG (1992, 1999). Os exemplares coletados como material de referência foram depositados nas coleções mastozoológicas da Universidade de Brasília e do Museu Nacional, Universidade Federal do Rio de Janeiro.

\section{RESULTADOS E DISCUSSÃO}

Foram obtidos registros de 85 espécies de mamíferos nativos no PNE e entorno (Tab. I), sendo que treze delas encontram-se na lista oficial de animais ameaçados de extinção (BERNARDES et al. 1990). O tipo de habitat de cada espécie, o número e a forma de registros que possibilitaram sua amostragem no PNE constam na tabela I. O sucesso de captura para pequenos mamíferos foi $5,5 \%$ para as armadilhas convencionais e $0,7 \%$ para armadilhas tipo "pitfall". O número de morcegos capturados foi de 126 . Segue um comentário geral sobre a lista, no que se refere aos vários grupos registrados. 
Tabela I. Lista das 85 espécies de Mamíferos observados no Parque Nacional das Emas, Goiás. Tipo de registros: (c) captura, (v) visualização. Habitat: (f) habitats fechados (floresta, cerradão e cerrado denso), (a) habitats abertos (campos, cerrado típico e cerrado ralo), sensu RibeIRo \& WALTER (1998). ( $\left.{ }^{\star}\right)$ Espécies listadas como ameaçadas (Bernardes et al. 1990), (\#) espécie encontrada apenas fora do PNE.

\begin{tabular}{lccc}
\hline Espécie & $\begin{array}{c}\text { Cariótipo } \\
(2 n / N F)\end{array}$ & Nome comum & Registros \\
\end{tabular}

Didelphimorphia

Didelphidae

Chironectes minimus (Zimmermann, 1780)

Didelphis albiventris Lund, 1840

Gracilinanus agilis (Burmeister, 1854)

Gracilinanus sp.

Lutreolina crassicaudata (Desmarest, 1804)

Marmosa murina (Linnaeus, 1758)

Monodelphis domestica (Wagner, 1842)

Monodelphis kunsi Pine, 1975

Philander opossum (Linnaeus 1758)

Thylamys sp.

Xenarthra

Myrmecophagidae

Myrmecophaga trydactyla Linnaeus, 1758 *

Tamandua tetradactyla (Linnaeus, 1758)

Dasypodidae

Cabassous unicinctus (Linnaeus, 1758)

Dasypus novemcinctus Linnaeus, 1758

Dasypus septemcinctus Linnaeus, 1758

Euphractus sexcinctus (Linnaeus, 1758)

Priodontes maximus (Kerr, 1792)*

Chiroptera

Phyllostomidae

Anoura caudifer (Geoffroy, 1818) \#

Anoura geoffroyi Gray, 1838)

Artibeus cinereus (Gervais, 1856) \#

Artibeus lituratus (Olfers, 1818)

Artibeus jamaicensis (Spix, 1823)

Carollia perspicillata (Linnaeus, 1758)

Chrotopterus auritus (Peters, 1856)

Desmodus rotundus (Geoffroy, 1810)

Glossophaga soricina (Pallas, 1766)

Lonchophylla dekeyseri Taddei et al., 1983

Micronycteris minuta (Gervais, 1856)

Phyllostomus discolor Wagner, 1843

Phyllostomus hastatus (Pallas, 1767) \#

Phyllostomus elongatus (Geoffroy, 1810) \#

Platyrrhinus lineatus (Geoffroy, 1810)

Sturnira lilium (Geoffroy, 1810)

Vespertilionidae

Eptesicus brasiliensis (Desmarest, 1819)

Lasiurus borealis (Müller, 1776)

Myotis sp.

Molossidae

Molossops planirostris (Peters, 1866)

Molossops temminckii (Burmeister, 1854)

Molossus molossus (Pallas, 1766)

Mormoopidae

Pteronotus gymnonotus Natterer 1843

Pteronotus parnellii Gray, 1843

Primates

Cebidae

Alouatta caraya (Humboldt, 1812)

Cebus apella (Linnaeus, 1758)

Cuica-d'água

Gambá, saruê

Mucura

Mucura

Cuíca

Mucura

Catita

Catita

Cuica-de-quatro-olhos

Mucura

1
43
4
4
4
20
2
1
2
1

Tamanduá-bandeira

Tamanduá-mirim, meleta

$\begin{array}{rcc}254 & v & f, a \\ 2 & v & f, a \\ 5 & v & a \\ 4 & v & f, a \\ 2 & v & f, a \\ 41 & v & a \\ 4 & v & a\end{array}$

Tatu-rabo-mole, ou rabo de couro

Tatu-verdadeiro, tatu-galinha

Tatu-china, tatu-galinha

Tatu-peba, tatu-peludo

Tatu-canastra

Morcego

Morcego

Morcego

Morcego

30

Morcego

Morcego

Morcego

28

Morcego-vampiro

Morcego

Morcego

Morcego

Morcego

Morcego

Morcego

Morcego

Morcego

Morcego

Morcego

Morcego

Morcego

Morcego

48

Morcego

Morcego

Morcego

Bugio, guariba

Macaco-prego $f, a$

f, a

$f$

$f, a$

$f$

a

f, a

f, a

a

t, a

a

a

f, a

f, a

f, a

f, a

f, a

f, a

f, a

f, a

f, a

f, a

f, a

f, a

f, a

i, a

f, a

f, a

f, a

f, a

f, a

f, a

f, a

f, a

f, a

f, a 
Tabela I. Continuação.

\begin{tabular}{|c|c|c|c|c|c|}
\hline \multirow{2}{*}{ Espécie } & \multirow{2}{*}{$\begin{array}{l}\text { Cariótipo } \\
\text { (2n/NF) }\end{array}$} & \multirow{2}{*}{ Nome comum } & \multicolumn{2}{|c|}{ Registros } & \multirow{2}{*}{ Habitat } \\
\hline & & & Número & Tipo & \\
\hline \multicolumn{6}{|l|}{ Carnivora } \\
\hline \multicolumn{6}{|l|}{ Canide } \\
\hline Cerdocyon thous (Linnaeus, 1766) & & Cachorro-do-mato & 42 & $v$ & $\mathrm{f}, \mathrm{a}$ \\
\hline Chrysocyon brachyurus (Illiger, 1815) * & & Lobo-guará & 80 & $v$ & a \\
\hline Pseudalopex vetulus (Lund, 1842) & & Raposa-do-campo & 52 & $v$ & a \\
\hline Speothos venaticus (Lund, 1842) * & & Cachorro-do-mato-vinagre & 1 & $v$ & $\mathrm{f}, \mathrm{a}$ \\
\hline \multicolumn{6}{|l|}{ Procyonidae } \\
\hline Nasua nasua (Linnaeus, 1766) & & Quati & 1 & v & $f$ \\
\hline Procyon cancrivorus (G. Cuvier, 1798) & & Mão-pelada & 10 & v & f, a \\
\hline \multicolumn{6}{|l|}{ Mustelidae } \\
\hline Conepatus semistriatus (Boddaert, 1784) & & Jaritataca & 65 & $v$ & a \\
\hline Eira barbara (Linnaeus, 1758) & & Irara, papa-mel & 3 & $v$ & $\mathrm{f}, \mathrm{a}$ \\
\hline Galictis cuja (Molina, 1782) & & Furão & 5 & $v$ & $f, a$ \\
\hline Lontra longicaudis (Olfers, 1818) * & & Lontra & 5 & v & $f$ \\
\hline \multicolumn{6}{|l|}{ Felidae } \\
\hline Herpailurus yaguarondi (Lacépède, 1809) & & Jaguarundi, gato-mourisco & 5 & $v$ & $\mathrm{f}, \mathrm{a}$ \\
\hline Leopardus pardalis (Linnaeus, 1758) * & & Jaguatirica & 2 & $v$ & $f$ \\
\hline Leopardus sp. * & & Gato-do-mato & 2 & $v$ & $f$ \\
\hline Oncifelis colocolo (Molina, 1810) * & & Gato-palheiro & 4 & $v$ & a \\
\hline Puma concolor (Linnaeus, 1771) * & & Sussuarana & 8 & v & $f, a$ \\
\hline Panthera onca (Linnaeus, 1758$)^{*}$ & & Onça-pintada & 1 & v & f, a \\
\hline Perissodactyla & & & & & \\
\hline \multicolumn{6}{|l|}{ Tapiridae } \\
\hline Tapirus terrestris (Linnaeus, 1758) & & Anta & 21 & $v$ & $\mathrm{f}, \mathrm{a}$ \\
\hline \multicolumn{6}{|l|}{ Artiodactyla } \\
\hline \multicolumn{6}{|l|}{ Tayassuidae } \\
\hline Pecari tajacu (Linnaeus, 1758) & & Caititu, cateto & 8 & $v$ & $\mathrm{f}, \mathrm{a}$ \\
\hline Tayassu pecari (Link, 1795) & & Queixada & 24 & $v$ & $\mathrm{f}, \mathrm{a}$ \\
\hline \multicolumn{6}{|l|}{ Cervidae } \\
\hline Blastocerus dichotomus (Illiger, 1815) * & & Cervo-do-pantanal & 2 & $v$ & a \\
\hline Mazama americana (Erxleben, 1777) & & Veado-mateiro & 2 & $v$ & $f_{1} a$ \\
\hline Mazama gouazoupira (G. Fischer, 1814) \# & & Veado-catingueiro & - & $v$ & f, a \\
\hline Ozotoceros bezoarticus (Linnaeus, 1758) * & & Veado-campeiro & 883 & $\mathrm{v}$ & a \\
\hline \multicolumn{6}{|l|}{ Rodentia } \\
\hline Muridae & & & & & \\
\hline Bolomys lasiurus (Lund, 1841) & 34 & Rato-do-mato & 354 & $\mathrm{c}$ & a \\
\hline Calomys expulsus (Lund, 1841) & & Rato-do-mato & 7 & $c$ & a \\
\hline Calomys tener (Winge, 1887) & & Rato-do-mato & 4 & $c$ & a \\
\hline Kunsia tomentosus (Lichtenstein, 1830) * & 44 & Rato-do-mato & 5 & c & a \\
\hline Nectomys squamipes (Brants, 1827) & 52 & Rato-d'água & 5 & c & $\mathrm{f}, \mathrm{a}$ \\
\hline Oecomys bicolor (Tomes, 1860 ) & $80 / 138$ & Rato-do-mato & 17 & $c$ & $f$ \\
\hline Oligoryzomys fornesi (Wagner, 1845) & $62 / 64$ & Rato-do-mato & 3 & $c$ & a \\
\hline Oryzomys gr. subflavus sp. 1 & $58 / 72$ & Rato-do-mato & 21 & $c$ & a \\
\hline Oryzomys gr. subflavus sp. 2 & 56 & Rato-do-mato & 11 & c & f \\
\hline Oryzomys megacephalus (Fischer, 1814) & 54 & Rato-do-mato & 35 & $c$ & $f$ \\
\hline Oxymycterus delator Thomas, 1903 & 54 & Rato-da-vereda & 23 & $c$ & a \\
\hline Pseudoryzomys simplex (Winge, 1887) \# & & Rato-do-mato & - & $c$ & a \\
\hline Erethizontidae & & & & & \\
\hline Coendou prehensilis (Linnaeus, 1758) & & Ouriço-cacheiro & 1 & v & f \\
\hline Caviidae & & & & & \\
\hline Cavia aperea Erxleben, 1777 & & Preá & 6 & $v$ & a \\
\hline Hydrochaeridae & & & & & \\
\hline Hydrochaeris hydrochaeris (Linnaeus, 1766) & & Capivara & 7 & v & f, a \\
\hline Dasyproctidae & & & & & \\
\hline Dasyprocta azarae Lichtenstein, 1823 & 62 & Cutia & $1 / 2$ & $c / v$ & f, a \\
\hline Agoutidae & & & & & \\
\hline Agouti paca (Linnaeus, 1766) & & Paca & 1 & $c$ & $f$ \\
\hline Echimyidae & & & & & \\
\hline Clyomys laticeps (Thomas, 1909) & 32 & Rato-de-espinho & 33 & $c$ & a \\
\hline Proechimys longicaudatus (Hengger, 1830) & 28 & Rato-de-espinho & 5 & c & $f$ \\
\hline
\end{tabular}


Ordem Didelphimorphia: registrou-se oito espécies de marsupiais, sendo Didelphis albiventris Lund, 1840 a mais comum, principalmente em ambientes florestais. Merecem destaque neste grupo o registro de uma espécie nova do gênero Gracilinanus (Gardner \& Creighton, 1989) e outra do gênero Thylamys (Gray, 1843), além da presença de Lutreolina crassicaudata (Desmarest, 1804) e Monodelphis kunsi Pine, 1975, animais pouco coletados e estudados (EMMONS \& FEER 1997). Outros marsupiais ainda são possíveis de serem encontrados, principalmente espécies que utilizam estratos superiores da vegetação, como as pertencentes ao gênero Caluromys (Allen, 1900), que dificilmente são capturadas em armadilhas ao nível do solo e do sub-bosque.

Ordem Xenarthra: tamanduá-bandeira (Myrmecophaga tridactyla (Linnaeus, 1758)) é uma das espécies de mamíferos mais observadas no Parque, ocorrendo em vários tipos de ambiente, especialmente próximo a campos úmidos. A principal ameaça para esta espécie no Parque são os grandes incêndios (SILVEIRA et al. 1999). O tamanduá-mirim (Tamandua tetradactyla (Linnaeus, 1758)) é bem mais raro e é encontrado principalmente em áreas de cerrado mais denso, apesar de também poder ser visto em campo aberto. Registrou-se cinco espécies de tatus, sendo Euphractus sexcinctus a mais comum. Dasypus novemcinctus Linnaeus, 1758 foi observado apenas nas proximidades do rio Formoso, enquanto D. septemcinctus Linnaeus, 1758, Cabassous unicinctus (Linnaeus, 1758) e Priodontes maximus (Kerr, 1792) ocorrem nas áreas mais secas. Rastros e tocas de tatu-canastra, $P$. maximus, são comuns, apesar da espécie ser raramente visualizada. REDFORD (1983) cita que Tolypeutes tricinctus (Linnaeus, 1758), o tatu-bola, era comum no Parque no passado. Há também relatos da ocorrência de tatus-bolas em regiões próximas ao Parque, a cerca de 20 anos atrás (A. Malheiros da Cruz, comunicação pessoal) e até hoje esta espécie é citada pelos moradores de Serranópolis, Goiás (cerca de 100 km do PNE). Porém, T. tricinctus foi só recentemente registrado no bioma do Cerrado (MARINHO-FILHO et al. 1997) e sua distribuição conhecida não abrange a região sudoeste do Estado de Goiás; portanto essas informações devem se referir a $T$. matacus (Desmarest, 1804), que ocorre nos Estados vizinhos, Mato Grosso e Mato Grosso do Sul (WeTZEL 1985a,b).

Ordem Chiroptera: registrou-se dezenove espécies de morcegos no Parque, porém um aumento no esforço de coleta deve acrescentar espécies à lista. Em outras áreas de Cerrado estudadas foram encontrados aproximadamente o mesmo número de espécies de morcegos, entre 16 (MARINHO-FILHO et al. 1998) e 25 espécies (WILlig 1983). A espécie mais coletada foi Molossus molossus (Pallas, 1766) (coletado próxima a construções), seguida por Platyrrhinus lineatus (Geoffroy, 1810). O morcego-vampiro Desmodus rotundus (Geoffroy, 1810) foi capturado principalmente na mata próxima à nascente do rio Jacuba, limite do Parque, provavelmente devido a proximidade com áreas de criação de gado, recurso alimentar utilizado pela espécie (TRAJANO 1996). São ainda merecedores de destaque os registros de Lonchophylla dekeyseri Taddei et al., 1983 (Phyllostomidae, Lonchophyllinae), único morcego endêmico do Cerrado (MARINHO-FILHO 1996), e de Chrotopterus auritus (Peters, 1856) e Pteronotus gymnonotus Natterer 1843, espécies raramente capturadas (MARINHO-FILHO et al. 1997, 1998; WILLIG 1983). 
Ordem Primates: duas espécies ocorrem no PNE: o bugio Alouatta caraya, raramente observado e o macaco-prego Cebus apella (Linnaeus, 1758), mais comum. Uma terceira espécie, Callithrix argentata (Linnaeus, 1771), é citada por REDFORD (1983), porém sem indicar a fonte da informação (se observação direta ou entrevista com terceiros). Nunca observou-se indícios de Callithrix (Erxleben, 1777) no Parque durante os cinco anos de estudo. Considerando que as espécies deste gênero são geralmente comuns e conspícuas nas áreas onde ocorrem, é improvável sua presença no PNE.

Ordem Carnívora: das 23 espécies de mamíferos carnívoros registradas para o Cerrado, 16 ocorrem no PNE, estando oito delas na lista oficial de mamíferos ameaçados de extinção. A Família Canidae é representada por quatro espécies: o lobo-guará Chrysocyon brachyurus (Illiger, 1815), a raposa-do-campo Pseudalopex vetulus (Lund, 1842), o cachorro-do-mato Cerdocyon thous (Linnaeus, 1766) e o cachorro-do-mato-vinagre Speothos venaticus (Lund, 1842). Dessas, as três primeiras são bastante comuns, e a quarta espécie muito rara, tendo sido observada apenas nove vezes nos últimos 33 anos (SILVEIRA et al. 1998). Pelo menos seis espécies da Família Felidae estão presentes no Parque, sendo que algumas, como a jaguatirica (Leopardus pardalis (Linnaeus, 1758)) e a onça-pintada (Panthera onça (Linnaeus, 1758)) são raras. O gato-mourisco (Herpailurus yaguarondi (Lácèpede, 1809)), a onça-parda (Puma concolor (Linnaeus, 1771)) e o gato-palheiro (Oncifelis colocolo (Molina, 1810)) são as espécies de felinos observadas mais comumente. Além da jaguatirica, pelo menos mais uma espécie de gato pintado ocorre no PNE, podendo ser Leopardus tigrinus (Schreber, 1775) e/ou L. wiedii (Schinz, 1821). Porém, ainda não se pôde ter a confirmação de qual das duas espécies. As duas espécies da Família Procyonidae que ocorrem no PNE, são: o quati (Nasua nasua (Linnaeus, 1766)), raramente observado, e o mão-pelada (Procyon cancrivorous (G. Cuvier, 1798)). Quatro espécies da Família Mustelidae foram registradas, sendo a jaritataca (Conepatus semistriatus (Boddaert, 1784)) a mais comum, sendo vista com frequiência nas estradas. O furão (Galictis cuja (Molina, 1782)), a lontra (Lontra longicaudis (Olfers, 1818)) e a irara (Eira bárbara (Linnaeus, 1758)) são mais raros. Uma quinta espécie, a ariranha (Pteronura brasiliensis (Gmelin, 1788)), é citada pelos guardaparque (REDFORD 1983), porém não conseguimos nenhuma evidência que comprove sua presença.

Ordem Perissodactyla: a anta (Tapirus terrestris (Linnaeus, 1758)) é uma espécie comum no Parque, sendo freqüentemente avistada. Seus rastros e fezes estão presentes em abundância em quase toda a área.

Ordem Artiodactyla: o veado-campeiro, Ozotoceros bezoarticus (Linnaeus, 1758), é o mamífero mais visualizado no PNE e um dos símbolos desta Unidade de Conservação. Existem cerca de 1.300 veados-campeiros no Parque (MERINO et al. 1997). Os outros cervídeos são bastante raros de se observar e restritos a ambientes de campo úmido, como o cervo-do-pantanal (Blastocerus dichotomus (Illiger, 1815)) ou de cerrado sensu stricto e matas, como o veado-mateiro (Mazama americana (Erxleben, 1777)) e o veado catingueiro (M. gouazoupira (G. Fischer, 1814)), este último observado apenas uma vez, no limite leste do Parque. Dentre os porcos do mato, o queixada (Tayassu pecari (Link, 1795)) é o mais comum, estando 
mais associado a áreas abertas e úmidas, enquanto o caititu (Pecari tajacu (Linnaeus, 1758)) é visto principalmente em habitats mais fechados, podendo também ser observado no campo.

Ordem Rodentia: registrou-se 20 espécies de roedores, distribuídos em sete famílias. A família Muridae é a mais numerosa, com 13 espécies, abrangendo ratos de hábitos variados, desde os semifossoriais e insetívoros, como Oxymycterus delator Thomas, 1903, a granívoros, como Bolomys lasiurus (Müller, 1776), e semi-aquáticos, como Nectomys squamipes (Brants, 1827). Dentre os roedores, B. lasiurus foi a espécie mais comum. Os principais os registros foram os de Kunsia tomentosus (Lichtenstein, 1830), animal raro e ameaçado de extinção, registrado pela primeira vez em uma Unidade de Conservação, e duas espécies novas de Oryzomys gr. subflavus, uma delas atualmente sendo descrita (CIBELE BONVICINO com. pess.) e a outra ainda não descrita. Existe também a possibilidade de os exemplares do gênero Clyomys (Thomas, 1916) pertencerem a uma espécie não conhecida pela ciência devido à diferença cariotípica com as outras duas espécies descritas para o gênero, C. laticeps (Thomas, 1909) e $C$. bishopi (Ávila-Pires \& Wutke, 1981), as quais apresentam o mesmo número diplóide $(2 n=34)$ e morfologia dos cromossomos (YONENAGA 1974; CIBELE BONVICINO com. pess.). Porém, até que se confirme o status taxonômico dos exemplares desta população, eles serão tratados como $C$. laticeps devido à semelhança em morfologia macroscópica aos caracteres diagnósticos desta espécie e à sua distribuição geográfica. A presença dos gêneros Kunsia Hershkovitz, 1966 e Clyomys, ambos de hábitos semifossoriais, já havia sido prevista por REDFORD (1983). A ratazana (Rattus rattus Linnaeus, 1758) foi registrada em residências no limite do PNE, mas não foi aqui incluída como parte da fauna do Parque.

\section{Análise da fauna}

O inventário apresentado neste estudo representa um acréscimo de $144 \%$ em relação à lista até então disponível (REDFORD 1983). Porém, é possível que várias espécies venham a ser acrescentadas à lista, especialmente na ordem Chiroptera, para qual o esforço de amostragem ainda deve ser aumentado. A fauna de mamíferos do PNE possui predominância de elementos de áreas abertas: $26,5 \%$ das espécies registradas ocupam os ambientes cerrado e campo, 19,2\% habitam florestas e 54,2\% ambos os ambientes. Em contraste, das 190 espécies de mamíferos registradas para o Cerrado, 28,2\% são habitantes de florestas, 55,3\% habitam tanto habitats florestados quanto os abertos e apenas 16,5\% são específicos de áreas abertas (MARINHOFILHO et al., no prelo).

Florestas de galeria são enclaves úmidos na região do Cerrado, permitindo que componentes faunísticos de outras regiões de vegetação florestal habitem este bioma e dispensando com isso a fauna local de desenvolver especializações a ambientes xéricos (REDFORD \& FONSECA 1986; MARINHO-FILHO \& REIS 1989). Por este motivo, a maior parte da fauna de mamíferos do Cerrado está associada, em maior ou menor grau, a ambientes florestados (FONSECA \& REDFORD 1984; REDFORD \& FONSECA 1986). Porém, 98\% dos habitats do PNE são de vegetação aberta (FERRONORTE 1998), explicando em parte, a menor representatividade de espécies florestais e também a baixa abundância de algumas espécies geralmente 
associadas a ambientes de vegetação mais densa, como o veado-mateiro (Mazama gouazoupira), a paca (Agouti paca (Linnaeus, 1766)), o quati (Nasua nasua) e morcegos de uma forma geral. Provavelmente pelo mesmo motivo outras espécies de ocorrência esperada pela sua distribuição geográfica não foram registradas, tais como os sagüis (Callithrix spp.) e o tapiti ou coelho-selvagem (Sylvilagus brasiliensis). O punaré, Thrichomys apereoides (Lund, 1841) (Rodentia, Echimyidae), também não foi encontrado no PNE, apesar de ser uma espécie de ampla distribuição, normalmente comum nas formações abertas do leste e do centro do Brasil (AlHo 1981; AlHo \& PEREIRA 1985; MOOJEN et al. 1988; DiETZ 1983) e associada a cerrados e campos. Por outro lado, espécies associadas a campos são muito abundantes, como o veado-campeiro (Ozotoceros bezoarticus), o tamanduá-bandeira (Myrmecophaga tridactyla), a jaritataca (Conepatus semistriatus) e o rato Bolomys lasiurus. O gato-palheiro, Oncifelis colocolo, espécie típica de habitats abertos e extremamente rara em outras localidades (OLIVEIRA 1994; SILVEIRA 1995), foi a segunda espécie de felino de pequeno porte mais observada no PNE.

Treze das dezessete espécies do Cerrado que constam da Lista Oficial do IBAMA da Fauna Brasileira Ameaçada de Extinção (BERNARDES et al. 1990) foram registradas no PNE (Tab. I). Uma destas, $K$. tomentosus, apesar de possuir uma área de distribuição ampla na América do Sul, é raramente encontrada e o PNE desempenha papel fundamental para a sua conservação, pois é a única reserva em que foi encontrada até o momento e uma das únicas unidades de conservação abrigando remanescentes da vegetação de campos abertos, ambiente onde a espécie ocorre e que se tornou extremamente raro na região sudoeste de Goiás, devido à expansão da fronteira agrícola. Apesar de estarem em uma Unidade de Conservação, ao menos duas das espécies novas encontradas (Gracilinanus sp. e Thylamys sp.) podem também ser consideradas ameaçadas, pois, com exceção da área do Parque, quase nada sobrou na região dos campos nativos onde esta espécie ocorre. As duas espécies novas de Oryzomys Baird, 1858 encontradas no PNE sofrem menos riscos, pois ocorrem em ambientes que podem ser encontrados mais freqüentemente fora do Parque: respectivamente em mata de galeria e todas as fisionomias de Cerrado, além de já terem sido encontradas em outras localidades (BONVICINO et al. 1999 e Cibele Bonvicino com. pess.).

Também para os mamíferos de maior porte, a fragmentação é o principal fator de risco para as populações. A fauna de mamíferos de maior porte apresenta maior mobilidade e consegue transitar entre o Parque e os fragmentos de Cerrado que ainda persistem no seu entorno. Estas áreas de vegetação nativa são fundamentais para a manutenção de fluxo gênico com outras populações, funcionando como corredores ecológicos ligando a região do PNE com outras áreas. Assim, o PNE tem conexão com o Pantanal, através do rio Taquari, que influencia as sub regiões Nhecolândia e Paiaguás do Pantanal (MMA 1999), com a Amazônia, através do rio Araguaia e com o rio Paranaíba (bacia do Paraná), através do rio Correntes, formado pela junção dos dois rios principais do PNE: o Jacuba e o Formoso. Porém, estes fragmentos sofrem constante pressão antrópica devido a desmatamento, uso para criação de gado, drenagem de áreas úmidas e ainda a ameaça de interrupção de corredores ecológicos por hidrelétricas. O principal exemplo é o corređor formado 
ao longo do rio Correntes devido a possível instalação da hidrelétrica de Itumirim. Estas áreas necessitam de atenção especial para que estes corredores não sejam interrompidos.

Devido à sua riqueza faunística, presença de espécies raras e ameaçadas de extinção e localização biogeográfica o PNE é uma das mais importantes Unidades de Conservação para a preservação de mamíferos no Cerrado. O Parque Nacional das Emas foi recentemente incluído nas Ações prioritárias para Conservação da Biodiversidade do Cerrado e Pantanal (MMA 1999) como área de importância biológica extremamente alta e representa uma área estratégica do Corredor Ecológico Cerrado/Pantanal. Dentre as ações prioritárias encontram-se a necessidade de efetivação de técnicas de manejo e criação de novas Unidades de Conservação no entorno, visando abranger áreas de vegetação mais densa distintas das encontradas no interior do PNE e conservar corredores ecológicos para a fauna do Parque. Inventários nos fragmentos de vegetação no entorno do PNE, em áreas com fitofisionomias densas, diferentes das encontradas no interior da Unidade, devem revelar a presença de outras espécies de mamíferos não registradas para o Parque, o que viria a ressaltar a importância destes fragmentos na conservação da diversidade biológica regional.

AGRADECIMENTOS. Somos gratos ao IBAMA pela autorização de pesquisa e pela infra-estrutura do Parque Nacional das Emas que nos foi oferecida. Em especial agradecemos a Ary Soares dos Santos, diretor do Parque na época do estudo, e aos funcionários Valdomiro, José Carlos, Nicássio e Heleno. Ao Dr. Jader S. Marinho-Filho pelo empréstimo de veículo para as viagens e discussões sobre o trabalho. À Cibele Bonvicino, por auxílio na identificação de alguns roedores e marsupiais. A todos os outros integrantes e ajudantes do projeto "Vertebrados do Parque Nacional das Emas" pelo auxílio no campo. Esse projeto recebeu apoio institucional da Fundação Ecológica de Mineiros - Fundação EMAS e apoio financeiro da Fundação O Boticário de Proteção à Natureza/MacArthur Foundation, BP Conservation/BirdLife International/Fauna \& Flora International, Wildlife Conservation Society - WCS e Conservation International do Brasil/ Heinhauser-Bush.

\section{REFERÊNCIAS BIBLIOGRÁFICAS}

ALHo, C.J.R. 1981. Small mammal populations of Brazilian Cerrado: the dependence of abundance and diversity on habitat complexity. Rev. Brasil. Biol. 41: 223-30.

ALHo, C.J.R. \& L.A. PEREIRA. 1985. Population ecology of a cerrado rodent community in central Brazil. Rev. Brasil. Biol. 45: 597-607.

Bernardes, A.T.; A.B.M. Machado \& A.B. Rylands. 1990. Fauna Brasileira Ameaçada de Extinção. Belo Horizonte, Fundação Biodiversitas, 65p.

Bonvicino, C. \& F.C. AlmeidA. 2000. Karyotype, morphology and taxonomic status of Calomys expulsus (Rodentia: Sigmodontinae). Mammalia 64 (3): 339-351.

Bonvicino, C.R., I. OtAZu \& P.M. Borodin. 1999. Chromosome variation in Oryzomys subflavus species group (Sigmodontinae, Rodentia) and its taxonomic implication. Cytologia 64: 327-332.

Bonvicino, C. \& M. WeKsLER. 1998. A new species of Oligoryzomys (Rodentia: Sigmodontinae) from northeastern and central Brazil. Z. Säugetierkunde 63: 90-103.

DiETZ, J.M. 1983. Notes on the natural history of some small mammals in central Brazil. Jour. Mammal. 64: 521-523. 
Emmons, L.H.\& F. Feer. 1997. Neotropical rainforest mammals. A field guide. Chicago, Univ. Chicago Press, $2^{\text {nd }}$ ed., XVI+307p.

ERIzE, F. 1977. Brazil's Finest National Park. Oryx 13: 457-462.

FErronorte. 1998. Plano Básico Ambiental (Trecho: Chapadão do Sul - Alto Taquari). São Paulo, FERRONORTE/TETRAPLAN, 150p.

FonSECA, G.A.B. \& K.H. REDFORD. 1984. The mammals of IBGE's Ecological Reserve, Brasília, and an analysis of the role of gallery forests in increasing diversity. Rev. Brasil. Biol. 44 (4): 517-523. IBDF/FBCN. 1981. Plano de Manejo do Parque Nacional das Emas. Brasília, IBDF/FBCN. 90p. MACEDO, J. 1995. Prospectives for the rational use of the Brazilian Cerrados for food production. Planaltina, EMBRAPA-CPAC, 19p.

MARINHO-FILHO, J.S. 1996. The Brazilian Cerrado Bat Fauna and its Conservation. Chiroptera Neotrop. 2 (1): 37-39.

Marinho-FiLho, J.S. \& M.L. ReIS. 1989. A fauna de mamíferos associada às matas de galeria, p. 43-60. In: L.M. BARbosA, (Ed.). Anais Simpósio sobre Mata Ciliar, Campinas, 335p.

Marinho-Filho, J.S.; M.M. Guimarães, M.L. Reis; F.H.G. Rodrigues; O. Torres \& G. Almeida. 1997. The discovery of Brazilian three banded armadillo in Cerrado of Central Brazil. Edentata 3 (1): 11-13.

Marinho-Filho, J.S.; D.C. Coelho \& F. Pinheiro. 1997. A comunidade de morcegos do Distrito Federal: Estrutura de guildas, uso do habitat e padrões reprodutivos, p. 123-126. In: L.L. LeITE \& C.H. SAITo (Eds). Contribuição ao conhecimento ecológico do Cerrado. Brasília, Univ. Brasília, $\mathrm{V}+325 \mathrm{p}$.

Marinho-Filho, J.S.; F.H.G. Rodrigues \& K.M. JuAREZ (no prelo). The Cerrado Mammals: diversity, ecology and natural history. In: P.S. OlIVEIRA, \& R.J. MARQUIS (Eds). The Cerrados of Brazil: ecology and natural history of a Neotropical savanna. Chicago, Chicago Univ. Press.

Marinho-Filho, J.S.; F.H.G. Rodrigues; M.M. Guimarães \& M.L. Reis. 1998. Os mamíferos da Estação Ecológica de Águas Emendadas, Planaltina, DF, p. 34-63. In: J. MARINHO-FILHO; F.H.G. Rodrigues \& M. GuIMARÃes (Eds).Vertebrados da Estação Ecológica de Águas Emendadas História Natural e Ecologia em um fragmento de cerrado do Brasil Central. Brasília, SEMATEC/IEMA, 92p.

Merino, M.L., S. Gonzales; F. Leeuwenberg; F.H.G. Rodrigues; L. Pinder \& W. M. TomÁs. 1997. Veado Campeiro (Ozotoceros bezoarticus Linnaeus. 1758). Distribuição, História Natural, Ecologia e Conservação, p. 42-58. In: J.M.B. DuARTE (Ed.). Biologia e Conservação de Cervídeos Sul-americanos: Blastocerus, Ozotoceros, e Mazama. Jaboticabal, FUNEP, 238p.

Mma. 1999. Ações Prioritárias para a Conservação da Biodiversidade do Cerrado e Pantanal. Brasília, Ministério do Meio Ambiente, 26p.

Moojen, J., S.F. ReIS \& M.V. Dellape. 1988. Quantitative variation in Thrichomys apereoides (Lund, 1841) (Rodentia, Echimyidae). I. Non-Geographic variation. Bol. Mus. Nac., n.s., Zool., Rio de Janeiro, 316: 1-15.

Musser, G.G.; M.D. Carleton; E.M. Brothers \& A.L. Gardner. 1998. Systematic studies of oryzomyine rodents (Muridae, Sigmodontinae): Diagnoses and distributions of species formerly assigned to Oryzomys “capito". Bull. Amer. Mus. Nat. Hist. 236: 1-376.

Nepstad, D.C.; C.A. KlinK; C. Uhl; I.C. Vieira; P. Lefebvre; M. Pedlowski; E. Matricardi; G. Negreiros; I. F. Brown; E. Amaral; A. Homma \& R. Walker. 1997. Land-use in Amazonia and the Cerrado of Brazil. Ci. Cult. 49 (1/2): 73-86.

Oliveira, T.G. 1994. Neotropical Cats: Ecology and Conservation. São Luís, Editora Univ. Federal do Maranhão, 244p.

PÁduA, M.T.J. 1996. Conservação in situ: unidades de conservação, p. 68-73. In: B.F.S. DiAs (Ed.). Alternativas de desenvolvimento dos cerrados: manejo e conservação dos recursos naturais renováveis. Brasília, Fundação Pró-Natureza, 97p.

REDFORD, K.H. 1983. Lista preliminar dos mamíferos do Parque Nacional das Emas. Brasil Florestal, Brasília, 55: 29-33. 
1985a. Emas National Park and the plight of the Brazilian Cerrados. Oryx 19 (4): 210-214. 1985b. Feeding and food preference in captive and wild giant anteaters (Myrmecophaga tridactyla). Jour. Zool. London 205 (4): 559-572. 1987. The pampas deer (Ozotoceros bezoarticus) in central Brazil, p. 410-414. In: C.M. WeMmer (Ed.) Biology and Management of the Cervidae. Washington, DC, Smithsonian Institution Press, XIII+577p.

REDFoRD, K.H. \& J.E. EISENBERG. 1992. Mammals of The Neotropics: The Southern Cone. Chicago, Chicago Univ. Press, 430p.

- 1999. Mammals of The Neotropics: The Central Neotropics. Chicago, Chicago Univ. Press, 609p.

REDFORD, K.H. \& G.A.B. FONSECA. 1986. The role of gallery forests in the zoogeography of the Cerrado's non-volant mammalian fauna. Biotropica 18 (2): 126-135.

Ribeiro, J.F. \& B.M.T. Walter. 1998. Fitofisionomias do bioma Cerrado, p. 89-166. In: S.M. Sano \& S.P. ALMEIDA (Eds). Cerrado: ambiente e flora. Planaltina, EMBRAPA-CPAC, XII+556p.

RodRIGUES, F.H.G. 1996. História Natural e Biologia Comportamental do Veado Campeiro no Parque Nacional das Emas. In: Anais XIV Encontro de Etologia, Uberlândia, p. 223-231.

Rodrigues, F.H.G. \& E.L.A. MonteIro-FilHo. 1997. Comensalistic interaction between the pampas deer (Ozotoceros bezoarticus, Mammalia, Cervidae) and rheas (Rhea americana, Aves, Rheidae). Brenesia 45/46: 187-188.

-1999. Feeding behaviour of the pampas deer: a grazer or a browser? Deer Specialist Group News, Philadelphia, 15: 12-13.

-2000. Home-range and activity of pampas deer in a brazilian cerrado. Jour. Mamm. 81 (4): 374-380.

Rodrigues, F.H.G.; L. Silveira; A.T. JACOMo \& E.L.A. MonteIro-Filho. 1999. Um albino parcial em veado campeiro, Ozotoceros bezoarticus bezoarticus, Artiodactyla, Cervidae. Revta bras. Zool. 16 (4): $1229-1232$.

SilveIrA, L. 1995. Notes on the distribution and natural history of the Pampas cat, Felis colocolo, in Brazil. Mammalia 59: 284-288.

Silveira, L.; A.T. JÁCOMO; F.H.G. Rodrigues \& P.G. CrawshaW JR. 1997. Hunting association between a aplomado falcon and maned wolf in Emas National Park, Central Brazil. Condor 99: 201-202.

Silveira, L.; A.T. JÁcomo; F.H.G. Rodrigues \& J.A. Diniz-Filho. 1998. Bush-dogs (Speothos venaticus) in Emas National Park, Central Brazil. Mammalia 62 (3): 446-449.

Silveira, L.; F.H.G. Rodrigues \& A.T. JÁcomo. 1999. Impact of Wildfires on The Megafauna of Emas National Park, Central Brazil. Oryx 33 (2): 108-114.

TRAJANO, E. 1996. Movements of cave bats in southeastern Brazil, with emphasis on the population ecology of the common vampire bat, Desmodus rotundus (Chiroptera). Biotropica 28: 121-129.

WETZEL, R.M. 1985a. The identification and distribution of recent Xenarthra (= Edentata), p. 5-21. In: G.G. MONTGOMERy (Ed.). The Evolution and Ecology of Armadillos, Sloths, and Vermilinguas. Washington, DC, Smithsonian Institution Press, 451p.

- 1985b. Taxonomy and distribution of armadillos, Dasypodidae, p. 23-46. In: G.G. Montgomery (Ed.). The Evolution and Ecology of Armadillos, Sloths, and Vermilinguas. Washington, DC, Smithsonian Institution Press, 451p.

WILLIG, M.R. 1983. Composition, microgeographic variation, and sexual dimorphism in Caatinga and Cerrado bat communities from Northeast Brazil. Bull. Carnegie Mus. Nat. Hist. 23:1-131.

WILSON, D.E. \& D.M. REEDER. 1993. Mammal species of the world - a taxonomic and geographic reference. Washington, Smithsonian Institution Press, $2^{\text {nd }}$ ed., XVIII+1207p.

WWF. 1999. Áreas protegidas ou espaços ameaçados? Série Técnica I, Brasília, WWF-Brasil, 17p.

YonenAGA, Y. 1975. Karyotypes and chromosome polymorphism in Brazilian rodents. Caryologia 28 (3): 269-286.

Recebido em 15.XII.2000; aceito em 21.V.2002.

Revta bras. Zool. 19 (2): $589-600,2002$ 